\title{
Estudio de mercado para cuyes de carne y reproductores, en el ámbito urbano de la provincia de Satipo
}

\section{Market for meat and breeding guinea pigs, in urban areas of the province of Satipo}

\author{
Bazán A. Luis / Laimes A. Celia / Palomino P. Guisela / Babilon A. Flor
}

Facultad de Ciencias Agrarias - Satipo, Universidad Nacional del Centro del Perú

\section{RESUMEN}

El objetivo del estudio fue determinar la población demandante y demanda insatisfecha en cuyes de carne y reproductores en el ámbito urbano de la provincia de Satipo, región Junín. La variables de análisis fueron población demandante de cuyes para carne y reproductores, oferta, demanda y el balance oferta demanda, tanto para cuyes de carne y reproductores. El estudio fue no experimental transeccional. Se realizo una muestreo estratificado en cada zona urbana de los diferentes distritos de la provincia de Satipo. La mayor proporción de habitantes que consumen carne de cuy fue en el distrito de Satipo, Rio Negro y Mazamari, siendo menor en los distritos de Pangoa y Rio Tambo. La población demandante de carne de cuy en el ámbito urbano de la provincia de Satipo, es de 40115.99 habitantes, y existe demanda insatisfecha para el consumo de carne de cuy en los habitantes del ámbito urbano de los distritos de la provincia de Satipo, siendo de 60370.22 cuyes para el año 2014, y proyectándose a 63764,35 para el año 2017. La población demandante de cuyes reproductores, en el ámbito urbano de la provincia de Satipo es de 13503.44 criadores, y existe demanda insatisfecha para proveer de cuyes reproductores a los criadores del ámbito urbano de los distritos de la provincia de Satipo, siendo 87787.03

\section{ABSTRACT}

The aim of the study was to determine the applicant and unmet demand for meat and breeding guinea pigs in urban areas of the province of Satipo, Junín region population. The analysis variables were applicant guinea pigs for meat and breeding, supply, demand and supply demand balance for both guinea pigs for meat and breeding population. The study was not experimental transeccional. Stratified one each urban area in different districts of the province of Satipo was collected. The largest proportion of residents who consume cuy meat was in the district of Satipo, Rio Black and Mazamari, being lower in districts Pangoa and Rio Tambo. The applicant of guinea pig meat in urban areas of the province of Satipo, population is 40115.99 inhabitants, and there is unmet demand for the consumption of guinea pig meat in the inhabitants of the urban areas of the districts of the province of Satipo, being of $60370.22 \mathrm{cu}$ yes 2014, and projected to 201763764.35 . spawning the applicant guinea pigs, in urban areas of the province of Satipo is 13503.44 breeders, and there is unmet demand for breeding guinea pigs to provide breeders urban areas of the districts of the province of Satipo, with players $87787.032014,92722.58$ and 2017 players. mentioned the hypothesis is accepted that if there applicant and unmet demand for meat and breeding guinea pigs, 
reproductores para el año 2014, y 92722.58 reproductores para el año 2017. Se acepta la hipótesis planteada que menciona que si existe población demandante y demanda insatisfecha en cuyes de carne y reproductores, en el ámbito urbano de la provincia de Satipo.

Palabras clave: cuyes, estudio de mercado, Satipo. in urban areas of the province of Satipo population.

Keywords: guinea pigs, market research, Satipo.

\section{INTRODUCCIÓN}

La selva por su biodiversidad es un ambiente en donde las crianzas familiares son un gran potencial para la seguridad alimentaria de los habitantes, tanto en la zona rural y urbana. Los migrantes de las zonas alto andinas hacia la selva, han traído su cultura y dentro de ello han logrado introducir y adaptar a estos climas cálidos al cuy. Chauca (2007) menciona que el cuy se desarrolla bien en climas templados, pudiendo adaptarse más a climas fríos que calientes y temperaturas superiores a $30^{\circ} \mathrm{C}$ los afectan pudiendo llegar a presentar stress de calor no pudiendo manejarse productivamente. Sin embargo en el ámbito de Satipo existiría una crianza y consumo considerable de cuy, sobre todo en habitantes migrantes y procedentes de migrantes. Jiménez (2007) menciona que durante la última década, tanto a nivel nacional como internacional se viene presentando una cada vez más creciente demanda por la carne de cobayo. Ordoñez (2003) menciona que la oferta actual de carne de cuy (140 TM anuales) cubre el $64 \%$ de la demanda (218 TM anuales), existiendo una demanda insatisfecha de 78 TM, también existe posibilidades de crecimiento del mercado actual pues un $28 \%$ de la población conoce el producto o es consumidor eventual y desearía hacerlo más seguido, bajo ciertas condiciones. Igualmente un $74 \%$ de la población es mercado potencial, comprendiendo a una población no consumidora pero abierta a un consumo futuro. En el ámbito de estudio, no existe un diagnóstico sobre la identificación de la población demandante, y de la demanda insatisfecha de cuyes para carne y sobre todo la necesidad de contar con reproductores en los criadores a nivel familiar. Este diagnóstico permitiría realizar planes de negocio o programas de apoyo, toda vez que los habitantes de la provincia de Satipo son muy dependientes de la venta de productos agrícolas, y la crianza familiar mejorada sería una alternativa en la diversificación de sus producciones. El objetivo del estudio fue determinar la población demandante y demanda insatisfecha en cuyes de carne y reproductores en el ámbito urbano de la provincia de Satipo. Los objetivos del estudio fueron:

- Determinar la población demandante y demanda insatisfecha para cuyes de carne, en el ámbito urbano de la provincia de Satipo.

- Determinar la población demandante y demanda insatisfecha para cuyes reproductores, en el ámbito urbano de la provincia de Satipo.

\section{MATERIAL Y MÉTODOS}

El trabajo de investigación se realizó en los ocho distritos de la provincia de Satipo, región Junín. El análisis de la información se realizó en la Facultad de Ciencias Agrarias de la Universidad Nacional del Centro del Perú, ubicado en el distrito de Río Negro. El diseño de la investigación fue no experimental, con enfoque cuantitativo Se tomó como población a 54284,16 habitantes del ámbito urbano de la provincia de Satipo (INEI-CPV 2007) y la muestra fue estratificada de las capitales de los ocho distritos de la provincia de Satipo, según criterio y recursos del investigador.

Variables de análisis:

1. Población demandante: Porcentaje de habitantes que consumen carne de cuy, del total 
de la población. Número de habitantes que consumen carne de cuy.

2. Balance oferta demanda para cuyes de carne: Oferta, Demanda.

3. Balance oferta demanda para cuyes reproductores: Oferta, Demanda

Para operacionalizar las variables de análisis se utilizó herramientas como son los cuestionarios, ejecutados con la técnica de entrevista personal para reunir información primaria. La entrevista estuvo dirigida al público de cada distrito sectorizado de acuerdo al índice demográfico de cada localidad. La estrategia de trabajo fue primeramente estructurar los instrumentos de recolección de información, capacitación y trabajo de campo. Para la recolección de información de campo se contó con el apoyo de alumnos de un Instituto Superior Tecnológico Público de la localidad. Las actividades realizadas fueron:

Los formatos del cuestionario, fueron elaborados teniendo en consideración la información que era necesaria obtener según las variables de análisis. Se realizaron tres tipos de encuestas, uno para determinar el análisis de la demanda, el segundo para determinar la oferta, y el tercero exclusivo para productores. Los cuestionarios según los objetivos planteados, fueron validados con la ayuda de un grupo de estudiantes en una prueba piloto.

\section{Zonas a intervenir:}

Las zonas a intervenir fueron las zonas urbanas porque dentro de ellos se encuentran el mayor flujo comercial de intercambio entre bienes y servicios, asimismo se escogió a las capitales de cada distrito. En la provincia de Satipo el 28\% de la población representa a la zona urbana.

Unidad de análisis: El grupo de estudio seleccionado para la investigación estuvo constituido por el total de personas que habitan en la zona urbana de las capitales de los distritos de la provincia de Satipo.

Población demandante: Se determinó la población demandante en base a un cuestionario donde se identificó el al porcentaje de pobladores que consume carne de cuy, y por otro lado al porcentaje de pobladores que realiza crianza familiar de cuy.

Demanda: Se realizó el análisis de la demanda actual, identificando el consumo per capita de carne de cuy y requerimiento de reproductores. Se determinó la proyección de la demanda de carne de cuy y reproductores.

Oferta: Se determinó la oferta de cuyes de carne y reproductores desde la perspectiva de crianzas familiares.

Balance Oferta demanda: Mediante el balance de la oferta y demanda se encontró la demanda insatisfecha en el ámbito de la zona urbana de la provincia de Satipo.

Estadísticos utilizados: Por ser un estudio descriptivo cuantitativo, se usó Tablas de doble entrada y distribución de frecuencia. El procesamiento de datos se realizó mediante el programa SPSS.

\section{RESULTADOS}

\section{POBLACIÓN DEMANDANTE}

La población demandante está determinada por el número de habitantes que consumen la carne de cuy en la provincia de Satipo. Se utilizó a la población de ámbito urbano, que han consumido carne de cuy, siendo siendo calculado en el $73.9 \%$ del total.

Tabla 1. Población demandante de carne de cuy estimada

\begin{tabular}{lrrrrrrrrr}
\hline \multirow{2}{*}{$\begin{array}{c}\text { Consumo de } \\
\text { carne de cuy }\end{array}$} & Satipo & Rio Negro & Mazamari & Pangoa & Llaylla & Coviriali & $\begin{array}{c}\text { Pampa } \\
\text { Hermosa }\end{array}$ & $\begin{array}{c}\text { Rio } \\
\text { Tambo }\end{array}$ & Satipo \\
\hline SI CONSUME \% & 82.6 & 90.9 & 78.3 & 57.1 & 64.7 & 88.2 & 90.0 & 46.7 & 73.9 \\
NO CONSUME \% & 17.4 & 9.1 & 21.7 & 42.9 & 35.3 & 11.8 & 10.0 & 53.3 & 26.1 \\
TOTAL \% & 100.0 & 100.0 & 100.0 & 100.0 & 100.0 & 100.0 & 100.0 & 100.0 & 100.0 \\
\hline
\end{tabular}

Fuente: INEI - CPV (2007), ZEE Satipo (2011) y elaboración propia (2014) 
Bazán A. Luis et al.

Tabla 2. Porcentaje estimado de la población que consume carne de cuy en los distritos de la provincia de Satipo.

\begin{tabular}{|c|c|c|c|c|c|c|c|c|c|c|}
\hline DETALLE & Satipo & Coviriali & Llaylla & Mazamari & $\begin{array}{c}\text { Pampa } \\
\text { Hermosa }\end{array}$ & Pangoa & Rio Negro & Rio Tambo & $\begin{array}{c}\text { Distrito } \\
\text { temporal }\end{array}$ & TOTAL \\
\hline Población total & 36307 & 5266 & 5143 & 28269 & 7508 & 29595 & 25981 & 32575 & 23228 & 193872 \\
\hline \% Población urbana & 68.82 & 3.45 & 20.16 & 33.49 & 6.64 & 31.1 & 5.91 & 5.47 & - & 28 \\
\hline Población urbana & 24986.48 & 181.68 & 1036.83 & 9467.29 & 498.53 & 9204.05 & 1535.48 & 1781.85 & - & 54284.16 \\
\hline $\begin{array}{l}\% \text { que consume carne } \\
\text { de cuy }\end{array}$ & 82.6 & 88.2 & 64.7 & 78.3 & 90 & 57.1 & 90.9 & 46.7 & - & 73.9 \\
\hline $\begin{array}{l}\text { Población } \\
\text { demandante de } \\
\text { Carne de cuy }\end{array}$ & 3999.99 & 605.3 & 419.57 & 3045.02 & 845.46 & 2130.24 & 3062.64 & 1789.06 & - & 40115.99 \\
\hline
\end{tabular}

Fuente: Elaboración propia (2014)

La mayor población demandante de carne de cuy fue para Satipo, seguido de Río Negro, Mazamari, Pangoa, Río Tambo, Pampa Hermosa, Coviriali y por ultimo Llaylla, haciendo un total de 40115.99 habitantes consumidores (población demandante) que representa el $73.9 \%$ del $100 \%$ de población urbana de los distritos de la provincia de Satipo, teniendo en cuenta que solo el $\mathbf{2 8 \%}$ de la población total de Satipo es del ámbito urbano (Cuadro 03).

\section{DEMANDA DE CARNE DE CUY}

Para la demanda de carne de cuy se realizo un análisis de los gustos y preferencias y el crecimiento poblacional.

\section{GUSTOS Y PREFERENCIAS:}

Como el ámbito de selva central, el consumo de carne es bastante limitada, lo cual es causado por el mal habito alimenticio de los pobladores que aun tienen basada la alimentación en el consumo de solo carbohidratos como es: yuca, plátano, papa, hortalizas y otros. Dentro del consumo local en carne tenemos a los siguientes:

\section{CRECIMIENTO POBLACIONAL}

Esta es una variable que tiene una influencia en la demanda del producto, la tendencia actual de los consumidores prefirieren los productos ecológicos, carne tierna, diferente al pollo o res. Según el estudio la población de Satipo consume la carne de cuy en un $73.9 \%$ principalmente porque gustan del producto.
Los pobladores de los distritos de Satipo quienes gustan o prefieren consumir carne de cuy, por ser exquisita, y de alto valor nutritivo. Es preciso mencionar que el numero de integrantes por familia con el que se esta trabajando es de 5 miembros.

A medida que la población va creciendo por efecto va haber una mayor demanda del producto, también hay que considerar la difusión e importancia del producto que cada día va siendo más conocido por las amas de casas y público en general.

\section{DETERMINACIÓN DE LA DEMANDA}

ACTUAL:

Población objetivo segmentada : 140116

Tasa de crecimiento : $\quad 1.84 \%$

Total personas encuestadas : 161

Tabla 3. Cantidad de animales que consumen al año

\begin{tabular}{|c|c|c|c|}
\hline $\begin{array}{l}19 \text { consumen } \\
42 \text { no consumen } \\
\text { Frecuencia de } \\
\text { consumo }\end{array}$ & $\begin{array}{l}\text { Total de } \\
\text { presas }\end{array}$ & $\begin{array}{l}\text { Total de } \\
\text { Animales }\end{array}$ & $\begin{array}{c}\text { Total } \\
\mathrm{kg}\end{array}$ \\
\hline Semanal & 1300 & 325 & 260 \\
\hline Mensual & 936 & 234 & 187.2 \\
\hline Anual & 119 & 29.75 & 23.8 \\
\hline Total & 2355 & 588.75 & 471 \\
\hline $\begin{array}{l}\text { Consumos } \\
\text { percapita }\end{array}$ & $\begin{array}{c}2.93 \mathrm{~kg} / \\
\text { habitante/ } \\
\text { año }\end{array}$ & & \\
\hline
\end{tabular}

Fuente: Elaboración propia (2014) 
Según nos muestra el cuadro; la demanda fue de $471 \mathrm{Kg}$ para una muestra de 161 personas, determinándose así un consumo percapita de $2.93 \mathrm{~kg}$ de carne de cuy/habitante/año, para la provincia de Satipo.

\section{PROYECCIÓN DE LA DEMANDA DE CARNE DE CUY}

Se determinó en base a la población total urbana multiplicada por el consumo per cápita. La demanda en $\mathrm{kg}$ y número de animales se observa a continuación:

Tabla 4. Demanda de la carne de cuy en el la provincia de Satipo

\begin{tabular}{ccccc}
\hline Año & $\begin{array}{c}\text { Población } \\
\text { total } \\
\text { Urbana* }\end{array}$ & $\begin{array}{c}\text { Consumo } \\
\text { percapita } \\
\text { Kg/hab/ } \\
\text { año }\end{array}$ & $\begin{array}{c}\text { Demanda } \\
\text { Carne de } \\
\text { cuy (kg) }\end{array}$ & $\begin{array}{c}N^{\circ} \text { de } \\
\text { cuyes }\end{array}$ \\
\hline 2007 & 54284.00 & 2.93 & 159052.12 & 198815.15 \\
2008 & 55282.83 & 2.93 & 161978.68 & 202473.35 \\
2009 & 56300.03 & 2.93 & 164959.09 & 206198.86 \\
2010 & 57335.95 & 2.93 & 167994.33 & 209992.92 \\
2011 & 58390.93 & 2.93 & 171085.43 & 213856.79 \\
2012 & 59465.32 & 2.93 & 174233.40 & 217791.75 \\
2013 & 60559.49 & 2.93 & 177439.30 & 221799.12 \\
2014 & 61673.78 & 2.93 & 180704.18 & 225880.22 \\
2015 & 62808.58 & 2.93 & 184029.14 & 230036.42 \\
2016 & 63964.26 & 2.93 & 187415.27 & 234269.09 \\
2017 & 65141.20 & 2.93 & 190863.71 & 238579.64 \\
\hline
\end{tabular}

Población urbana $28 \%$ de la población total

Fuente: Elaboración propia (2014), según datos del INEI (2007)

\section{OFERTA DE LA CARNE DE CUY}

No existe a nivel de la zona empresas que se dediquen a la crianza comercial de cuyes permanentemente, debido a diversos factores sociopolíticos, estabilidad económica y problemas del proceso productivo hasta el nivel de comercialización, que hace que los inversionistas puedan hacer empresa.

La metodología a emplearse es la misma que se utilizó en el estudio de la demanda, aquí también no existen registros históricos en instituciones. Los datos que proporcionamos en el estudio han sido hallados utilizando la metodología de entrevista y observación directa. En la actualidad la oferta del producto que es la carne de cuy siendo abastecido por solo por aquellos productores de la zona, y solo se realiza de manera eventual.

Por otra parte existieron en años anteriores algunos proyectos ejecutados por instituciones no gubernamentales con el objetivo de fomentar la crianza de cuyes, pero debido a múltiples factores de monitoreo, no prosperaron aquellos proyectos.

\section{PRINCIPALES ZONAS PRODUCTORAS:}

En la actualidad en el ámbito de Satipo no se cuenta con zonas exitosas de producción de cuyes, los únicos distritos que han implementado sus centros de producción en cuyes a través de proyectos de inversión pública son Satipo y Coviriali, que a la fecha tienen instalados, dos centros de producción de cuyes con una capacidad instalada total de 115 y 150 reproductores respectivamente, y su producción es para abastecer a los beneficiarios de dichos proyectos.

\section{CAPACIDAD DE PRODUCCIÓN SEGÚN DIS- TRITOS:}

Como se detallo en el párrafo anterior no existe una producción a nivel comercial solo por algunos productores que llegan a tener una población de $10-200$ animales, con un numero de vientres entre 5 a 35 animales, a continuación detallaremos la capacidad instalada por los productores.

\section{PRINCIPALES ABASTECEDORES DE CARNE DE CUY}

Los principales abastecedores de carne de cuy son los mismos productores de crianza familiar en Satipo, no existen empresas formales que se dediquen a la producción de cuy en gran escala. Sin embargo existen pequeños productores como la familia Rivera que realiza crianza familiar comercial y expende en su propio centro recreacional ubicada en el Centro poblado 
Zanjohuatziari a 15 minutos de zona urbana de Satipo, contando con 50 vientres aproximadamente. Entonces como ya se menciono no existe abastecedor de carne de cuy, pero es importante mencionar que los fines de semana existe ingreso de cuy de la zona de Tarma, Huancayo y Lima para las festividades y actividades sociales. Existen personas comercializadoras de cuyes en Satipo, quienes acopian y revenden después de un periodo de engorde.

Tabla 5. Porcentaje de familias dedicadas a la crianza familiar en Satipo

\begin{tabular}{lrrrrrrrrr}
\hline & \multicolumn{7}{c}{ Distritos de Satipo } & & Total \\
\cline { 2 - 10 } & Satipo & Rio Negro & Mazamari & Pangoa & \multicolumn{1}{c}{ Llaylla } & Coviriali & $\begin{array}{c}\text { Pampa } \\
\text { Hermosa }\end{array}$ & Rio Tambo & Satipo \\
\cline { 2 - 10 } SI \% & 43.5 & 36.4 & 60.9 & 28.6 & 41.2 & 82.4 & 30.0 & 6.7 & 41.0 \\
NO \% & 56.5 & 63.6 & 39.1 & 71.4 & 58.8 & 17.6 & 70.0 & 93.3 & 59.0 \\
TOTAL \% & 100.0 & 100.0 & 100.0 & 100.0 & 100.0 & 100.0 & 100.0 & 100.0 & 100.0 \\
\hline
\end{tabular}

Fuente: Elaboración propia (2014)

Tabla 6. Destino de los cuyes de las crianzas familiares en Satipo

\begin{tabular}{|c|c|c|c|c|c|c|c|c|c|}
\hline & \multicolumn{8}{|c|}{ Distritos de Satipo } & \multirow{2}{*}{$\begin{array}{l}\text { Total } \\
\text { Satipo }\end{array}$} \\
\hline & Satipo & $\begin{array}{c}\text { Rio } \\
\text { Negro }\end{array}$ & Mazamari & Pangoa & Llaylla & Coviriali & $\begin{array}{l}\text { Pampa } \\
\text { Hermosa }\end{array}$ & $\begin{array}{c}\text { Rio } \\
\text { Tambo }\end{array}$ & \\
\hline Venta para carne (\%) & 20.0 & 50.0 & 21.4 & 100.0 & 28.6 & 12.5 & 33.3 & .0 & 23.1 \\
\hline $\begin{array}{l}\text { Venta para } \\
\text { reproductores (\%) } \\
\text { Venta para carne y }\end{array}$ & 3.3 & .0 & .0 & .0 & .0 & .0 & .0 & .0 & 1.3 \\
\hline $\begin{array}{l}\text { reproductores } \\
(\%)\end{array}$ & 13.3 & .0 & 7.1 & .0 & 71.4 & 50.0 & .0 & .0 & 23.1 \\
\hline $\begin{array}{l}\text { Exclusivo para } \\
\text { autoconsumo (\%) }\end{array}$ & 33.3 & 50.0 & 71.4 & .0 & .0 & 25.0 & 66.7 & 100.0 & 38.5 \\
\hline No cría cuyes (\%) & 30.0 & .0 & .0 & .0 & .0 & 12.5 & .0 & .0 & 14.1 \\
\hline Total ( \%) & 100.0 & 100.0 & 100.0 & 100.0 & 100.0 & 100.0 & 100.0 & 100.0 & 100.0 \\
\hline
\end{tabular}

\section{OFERTA DE LA CARNE DE CUY}

En el caso de la oferta del producto (carne de cuy), está orientado básicamente al mercado local, donde la comercialización se realiza en venta directa al consumidor o por otra parte también se venden a algunos restaurantes turísticos o pedidos en épocas de festividades, estas ultimas son abastecidas por granjas ubicadas fuera de a provincia y en su mayoría por intermediarios.

La oferta de cuyes para carne se obtuvo a partir del estimado de la venta mensual y anual de los criadores bajo modalidad familiar, siendo 200 y 2400 cuyes respectivamente para una muestra de 161 entrevistados. Las familias se estimo en base a la población urbana, con un número de 5 integrantes por familia, de los cuales el $41 \%$ se dedica a la crianza de cuyes a nivel familiar.

\section{PROYECCIÓN DE LA OFERTA}

Tabla 7. Oferta de cuyes para carne de crianzas familiares en Satipo

\begin{tabular}{ccccc}
\hline Año & Familias & $\begin{array}{c}\% \\
\text { Familias } \\
\text { criadoras }\end{array}$ & $\begin{array}{c}\text { Criadores } \\
\text { familiares }\end{array}$ & $\begin{array}{c}\mathrm{N}^{\circ} \text { cuyes } \\
\text { (carne) }\end{array}$ \\
\hline 2007 & 10857 & 41 & 4451 & 145678.52 \\
2008 & 11057 & 41 & 4533 & 148359.00 \\
2009 & 11260 & 41 & 4617 & 151088.81 \\
2010 & 11467 & 41 & 4702 & 153868.84 \\
2011 & 11678 & 41 & 4788 & 156700.03 \\
2012 & 11893 & 41 & 4876 & 159583.31 \\
2013 & 12112 & 41 & 4966 & 162519.64 \\
2014 & 12335 & 41 & 5057 & 165510.00 \\
2015 & 12562 & 41 & 5150 & 168555.39 \\
2016 & 12793 & 41 & 5245 & 171656.81 \\
2017 & 13028 & 41 & 5342 & 174815.29 \\
\hline
\end{tabular}

Fuente: Elaboración propia (2014) 


\section{BALANCE OFERTA DEMANDA DE LA CARNE DE CUY}

Según el estudio se puede apreciar que existe una brecha por cubrir o demanda insatisfecha para los cuyes de carne siendo de 60370,22 animales en el año 2014.

Tabla 8. Balance de la Oferta y Demanda de cuyes para carne en la provincia de Satipo

\begin{tabular}{cccc}
\hline Año & $\begin{array}{c}\text { Oferta } \\
\mathrm{N}^{\circ} \text { cuyes } \\
\text { Carne }\end{array}$ & $\begin{array}{c}\text { Demanda } \\
\mathrm{N}^{\circ} \text { cuyes } \\
\text { carne }\end{array}$ & $\begin{array}{c}\text { Demanda } \\
\text { insatisfecha } \\
\text { cuyes carne }\end{array}$ \\
\hline 2007 & 145678.52 & 198815.15 & -53136.63 \\
2008 & 148359.00 & 202473.35 & -54114.35 \\
2009 & 151088.81 & 206198.86 & -55110.05 \\
2010 & 153868.84 & 209992.92 & -56124.08 \\
2011 & 156700.03 & 213856.79 & -57156.76 \\
2012 & 159583.31 & 217791.75 & -58208.44 \\
2013 & 162519.64 & 221799.12 & -59279.48 \\
2014 & 165510.00 & 225880.22 & -60370.22 \\
2015 & 168555.39 & 230036.42 & -61481.03 \\
2016 & 171656.81 & 234269.09 & -62612.28 \\
2017 & 174815.29 & 238579.64 & -63764.35 \\
\hline
\end{tabular}

Fuente: Elaboración propia (2014)

POBLACION DEMANDANTE DE RE-

\section{PRODUCTORES}

Del total de entrevistados el $41 \%$ manifestó que se dedica a la crianza familiar de cuyes, los cuales lo realizan en un $82.1 \%$ con cuyes cruzados que no han sido mejorados genéticamente, por tanto obtienen bajos índices productivos, y tienen la necesidad de adquirir reproductores mejorados, teniendo así un gran mercado.

Tabla 9. Población demandante de reproductores en la provincia de Satipo

\begin{tabular}{lr}
\hline \multicolumn{1}{c}{ DETALLE } & \multicolumn{1}{c}{ TOTAL } \\
\hline Población total & 193872 \\
$\%$ Población urbana & 28 \\
Población urbana & 54284.16 \\
$\%$ que consume carne & 73.9 \\
de cuy & \\
$\begin{array}{l}\text { Población demandante } \\
\text { de carne de cuy }\end{array}$ & 40115.99 \\
$\begin{array}{l}\text { Población que se dedica } \\
\text { a crianza de cuy }\end{array}$ & 16447.56 \\
$\begin{array}{l}\text { Población que trabaja } \\
\text { con cuyes cruzados }\end{array}$ & 13503.44 \\
$\begin{array}{l}\text { Población demandante } \\
\text { de reproductores }\end{array}$ & 13503.44 \\
\hline $\begin{array}{l}\text { Fuente: INEI - CPV (2007), ZEE Satipo (2011) y elaboración } \\
\text { propia (2014) }\end{array}$ & \\
DEMANDA DE REPRODUCTORES
\end{tabular}

DEMANDA DE REPRODUCTORES

Tabla 10. Familias que se dedican a la crianza familiar de cuyes en los distritos de Satipo

\begin{tabular}{|c|c|c|c|c|c|c|c|c|c|}
\hline & \multicolumn{8}{|c|}{ Distritos de Satipo } & \multirow{2}{*}{$\begin{array}{l}\text { Total } \\
\text { Satipo }\end{array}$} \\
\hline & Satipo & $\begin{array}{c}\text { Rio } \\
\text { Negro }\end{array}$ & Mazamari & Pangoa & Llaylla & Coviriali & $\begin{array}{c}\text { Pampa } \\
\text { Hermosa }\end{array}$ & $\begin{array}{c}\text { Rio } \\
\text { Tambo }\end{array}$ & \\
\hline SI \% & 43.5 & 36.4 & 60.9 & 28.6 & 41.2 & 82.4 & 30.0 & 6.7 & 41.0 \\
\hline NO \% & 56.5 & 63.6 & 39.1 & 71.4 & 58.8 & 17.6 & 70.0 & 93.3 & 59.0 \\
\hline Total \% & 100.0 & 100.0 & 100.0 & 100.0 & 100.0 & 100.0 & 100.0 & 100.0 & 100.0 \\
\hline
\end{tabular}

Fuente: Elaboración propia (2014). 
Tabla 11. Líneas de cuyes de las familias criadoras de cuyes en Satipo

\begin{tabular}{|c|c|c|c|c|c|c|c|c|c|}
\hline & \multicolumn{8}{|c|}{ Distritos de Satipo } & \multirow{2}{*}{$\begin{array}{l}\text { Total } \\
\text { Satipo }\end{array}$} \\
\hline & Satipo & Rio Negro & Mazamari & Pangoa & Llaylla & Coviriali & $\begin{array}{c}\text { Pampa } \\
\text { Hermosa }\end{array}$ & $\begin{array}{c}\text { Rio } \\
\text { Tambo }\end{array}$ & \\
\hline MEJORADOS \% & 33.3 & 25.0 & .0 & .0 & .0 & 28.6 & .0 & $.0 \%$ & $17.9 \%$ \\
\hline CRUZADOS \% & 66.7 & 75.0 & 100.0 & 100.0 & 100.0 & 71.4 & 100.0 & $100.0 \%$ & $82.1 \%$ \\
\hline Total \% & 100.0 & 100.0 & 100.0 & 100.0 & 100.0 & 100.0 & 100.0 & $100.0 \%$ & $100.0 \%$ \\
\hline
\end{tabular}

Fuente: Elaboración propia (2014)

Tabla 12. Requerimiento de cuyes reproductores mejorados Satipo

\begin{tabular}{ccccccccccc}
\hline & \multicolumn{1}{c}{ Distritos de Satipo } & & Total \\
\cline { 2 - 9 } & Satipo & Rio Negro & Mazamari & Pangoa & Llaylla & Coviriali & $\begin{array}{c}\text { Pampa } \\
\text { Hermosa }\end{array}$ & Rio Tambo & Satipo \\
\hline SI \% & 100.0 & 75.0 & 28.6 & 100.0 & 100.0 & 92.9 & 100.0 & 100.0 & 82.1 \\
NO $\%$ & .0 & 25.0 & 71.4 & .0 & .0 & 7.1 & .0 & .0 & 17.9 \\
TOTAL $\%$ & 100.0 & 100.0 & 100.0 & 100.0 & 100.0 & 100.0 & 100.0 & 100.0 & 100.0 \\
\hline Fuente: Elabo
\end{tabular}

Fuente: Elaboración propia (2014)

Tabla 13. Demanda de reproductores hembra en las familias criadoras de cuyes en la provincia de Satipo.

\begin{tabular}{ccccc}
\hline Año & Familias & Porcentaje & $\begin{array}{c}\text { Criadores } \\
\text { familiares }\end{array}$ & $N^{\circ}$ de cuyes \\
\hline 2007 & 10857 & 0.41 & 4451 & 57507.32 \\
2008 & 11057 & 0.41 & 4533 & 58565.46 \\
2009 & 11260 & 0.41 & 4617 & 59643.06 \\
2010 & 11467 & 0.41 & 4702 & 60740.49 \\
2011 & 11678 & 0.41 & 4788 & 61858.12 \\
2012 & 11893 & 0.41 & 4876 & 62996.31 \\
2013 & 12112 & 0.41 & 4966 & 64155.44 \\
2014 & 12335 & 0.41 & 5057 & 65335.90 \\
2015 & 12562 & 0.41 & 5150 & 66538.08 \\
2016 & 12793 & 0.41 & 5245 & 67762.38 \\
2017 & 13028 & 0.41 & 5342 & 69009.21 \\
\hline
\end{tabular}

Fuente: Elaboración propia (2014)
Tabla 14 Demanda de reproductores macho en las familias criadoras de cuyes en la provincia de Satipo.

\begin{tabular}{ccccc}
\hline Año & Familias & Porcentaje & $\begin{array}{c}\text { Criadores } \\
\text { familiares }\end{array}$ & $N^{\circ}$ de cuyes \\
\hline 2007 & 10857 & 0.41 & 4451 & 24414.64 \\
2008 & 11057 & 0.41 & 4533 & 24863.87 \\
2009 & 11260 & 0.41 & 4617 & 25321.36 \\
2010 & 11467 & 0.41 & 4702 & 25787.28 \\
2011 & 11678 & 0.41 & 4788 & 26261.76 \\
2012 & 11893 & 0.41 & 4876 & 26744.98 \\
2013 & 12112 & 0.41 & 4966 & 27237.09 \\
2014 & 12335 & 0.41 & 5057 & 27738.25 \\
2015 & 12562 & 0.41 & 5150 & 28248.63 \\
2016 & 12793 & 0.41 & 5245 & 28768.41 \\
2017 & 13028 & 0.41 & 5342 & 29297.75 \\
\hline
\end{tabular}

Fuente: Elaboración propia (2014) 
Tabla 15. Demanda de reproductores hembras y machos en las familias criadoras de cuyes en la provincia de Satipo.

\begin{tabular}{ccccc}
\hline Año & Familias & Porcentaje & $\begin{array}{c}\text { Criadores } \\
\text { familiares }\end{array}$ & $N^{\circ}$ de cuyes \\
\hline 2007 & 10857 & 0.41 & 4451 & 81921.96 \\
2008 & 11057 & 0.41 & 4533 & 83429.33 \\
2009 & 11260 & 0.41 & 4617 & 84964.43 \\
2010 & 11467 & 0.41 & 4702 & 86527.77 \\
2011 & 11678 & 0.41 & 4788 & 88119.88 \\
2012 & 11893 & 0.41 & 4876 & 89741.29 \\
2013 & 12112 & 0.41 & 4966 & 91392.53 \\
2014 & 12335 & 0.41 & 5057 & 93074.15 \\
2015 & 12562 & 0.41 & 5150 & 94786.72 \\
2016 & 12793 & 0.41 & 5245 & 96530.79 \\
2017 & 13028 & 0.41 & 5342 & 98306.96 \\
\hline
\end{tabular}

Fuente: Elaboración propia (2014)

\section{OFERTA DE CUYES PARA REPRODUC- TORES}

Se obtuvo a partir del estimado de la venta mensual y anual de los criadores bajo modalidad familiar, siendo 69 y 828 cuyes respectivamente para una muestra de 161 entrevistados. Las familias se estimó en base a la población urbana, con un número de 5 integrantes por familia, de los cuales el $41 \%$ se dedica a la crianza de cuyes a nivel familiar.

Tabla 16. Oferta de cuyes para reproductores provenientes de crianzas familiares en Satipo

\begin{tabular}{ccccc}
\hline Año & Familias & $\begin{array}{c}\text { \% Familias } \\
\text { Criadoras }\end{array}$ & $\begin{array}{c}\text { Criadores } \\
\text { familiares }\end{array}$ & $\begin{array}{c}\mathrm{N}^{\circ} \text { cuyes } \\
\text { (reproductores) }\end{array}$ \\
\hline 2007 & 10857 & 0.41 & 4451 & 4653.62 \\
2008 & 11057 & 0.41 & 4533 & 4739.25 \\
2009 & 11260 & 0.41 & 4617 & 4826.45 \\
2010 & 11467 & 0.41 & 4702 & 4915.25 \\
2011 & 11678 & 0.41 & 4788 & 5005.70 \\
2012 & 11893 & 0.41 & 4876 & 5097.80 \\
2013 & 12112 & 0.41 & 4966 & 5191.60 \\
2014 & 12335 & 0.41 & 5057 & 5287.13 \\
2015 & 12562 & 0.41 & 5150 & 5384.41 \\
2016 & 12793 & 0.41 & 5245 & 5483.48 \\
2017 & 13028 & 0.41 & 5342 & 5584.38 \\
\hline
\end{tabular}

Fuente: Elaboración propia (2014)

\section{BALANCE OFERTA DEMANDA PARA CUYES REPRODUCTORES}

Según el estudio se puede apreciar que existe una brecha por cubrir o demanda insatisfecha para los cuyes de carne siendo de 84643.49 reproductores en el año 2012, lo cual garantiza el mercado del negocio con miras a ofrecer reproductores mejorados de calidad y sobre todo adaptados al clima tropical.

Tabla 17. Balance de la Oferta y Demanda de cuyes para reproductores en la provincia de Satipo

\begin{tabular}{cccc}
\hline Año & $\begin{array}{c}\text { Oferta } \\
N^{\circ} \text { cuyes } \\
\text { Reproductores }\end{array}$ & $\begin{array}{c}\text { Demanda } \\
N^{\circ} \text { cuyes } \\
\text { reproductores }\end{array}$ & $\begin{array}{c}\text { Demanda } \\
\text { insatisfecha } \\
\text { cuyes } \\
\text { reproductores }\end{array}$ \\
\hline 2007 & 4653.62 & 81921.96 & -77268.34 \\
2008 & 4739.25 & 83429.33 & -78690.08 \\
2009 & 4826.45 & 84964.43 & -80137.98 \\
2010 & 4915.25 & 86527.77 & -81612.52 \\
2011 & 5005.70 & 88119.88 & -83114.19 \\
2012 & 5097.80 & 89741.29 & -84643.49 \\
2013 & 5191.60 & 91392.53 & -86200.93 \\
2014 & 5287.13 & 93074.15 & -87787.03 \\
2015 & 5384.41 & 94786.72 & -89402.31 \\
2016 & 5483.48 & 96530.79 & -91047.31 \\
2017 & 5584.38 & 98306.96 & -92722.58 \\
\hline
\end{tabular}

Fuente: Elaboración propia (2014)

\section{DISCUSIÓN}

La carne de cuy se encuentra posesionada en el mercado como una carne muy exquisita, típica de entre los platos de la sierra, resultado objetivamente verificable en los recreos y restaurantes de la sierra en las que figura el tradicional y famoso "picante de cuy" como un plato obligatorio. Tradición que se ha venido posesionando con mucha fuerza en la costa e inclusive en la selva de nuestro país, siendo el poblador andino, el que consume con mayor frecuencia en comparación con el poblador de la costa y la selva. 
No existe ningún dato estadístico sobre el consumo de la carne de cuyes, ni estimaciones referenciales sobre el consumo per-cápita, debido a que su consumo tiene características muy particulares.

El consumo en la población rural en la sierra es cotidiano, mientras que en la población urbana, su consumo es muy limitado en sectores de clase media y aun en este, muy restringido, pues solamente lo hacen en fechas especiales, como los cumpleaños y fiestas especiales. La carne de cuy es cara en relación con las demás especies y se mantiene invariable por muchos años.

El $73.9 \%$ de los habitantes del ámbito urbano de Satipo consume carne de cuy, este valor es superior al encontrado por Ordoñez (2003) quien menciona que se puede concluir que un $44.2 \%$ de amas de casa de Lima son consumidores eventuales o frecuentes de la carne de cuy y que, adicionalmente, un $29.5 \%$ de ellas, es proclive a serlo, sumando en total $73.7 \%$ de la población como mercado potencial.

El consumo percapita de $2.93 \mathrm{~kg}$ de carne de cuy/habitante/año, para la provincia de Satipo, equivalente a $0.28 \mathrm{~kg} / \mathrm{semana} / \mathrm{familia}$, es inferior $0.83 \mathrm{~kg}$ obtenido por la Municipalidad de Kimbiri, citado por Machahuay (2013).

La carne de cuy ha mostrado una tendencia creciente en los últimos años producto de la promoción de la carne y la migración de familias de la provincia hacia la ciudad, que mantienen sus costumbres, tradiciones y hábitos de consumo del producto carcasa del cuy.

En cuanto al consumo de carne de cuy, se puede observar la existencia de una demanda insatisfecha no cubierta, esto permite visualizar una sostenibilidad de largo plazo para una producción de cuyes, adicional que excede largamente las máximas pretensiones de proyectos de similar envergadura que el presente, se necesitaría por lo menos de 5820 hembras reproductoras para cubrir esta demanda, establecidas en galpones en diferentes zonas con potencialidades para cubrir la demanda insatisfecha. Se requiere 84643.49 reproductores en la provincia de Satipo para abastecer la demanda insatisfecha de reproductores mejorados, considerado en cada distrito 1058 reproductores, siendo necesario de al menos 1 centro piloto con 200 vientres por cada distrito.

\section{CONCLUSIONES}

- La población demandante de carne de cuy en el ámbito urbano de la provincia de Satipo, es de 40115.99 habitantes, y existe demanda insatisfecha para el consumo de carne de cuy en los habitantes del ámbito urbano de los distritos de la provincia de Satipo, siendo de 60370.22 cuyes para el año 2014, y proyectándose a 63764.35 para el año 2017.

- La población demandante de cuyes reproductores, en el ámbito urbano de la provincia de Satipo es de 13503.44 criadores, y existe demanda insatisfecha para proveer de cuyes reproductores a los criadores del ámbito urbano de los distritos de la provincia de Satipo, siendo 87787.03 reproductores para el año 2014 y 92722.58 reproductores para el año 2017.

- Se acepta la hipótesis planteada que menciona que si existe población demandante y demanda insatisfecha en cuyes de carne y reproductores, en el ámbito urbano de la provincia de Satipo.

\section{RECOMENDACIONES}

- Implementar Proyectos de producción de cuyes con énfasis a carne y reproductores en los distritos de la provincia de Satipo.

- Realizar estudios similares en el ámbito rural de la provincia de Satipo.

- Proponer un programa de mejoramiento animal para cuyes adaptados al ecosistema tropical de selva alta.

\section{REFERENCIAS BIBLIOGRÁFICAS}

1. Jiménez Aliaga, Ronald (2007). Uso de insumos agrícolas locales en la alimentación de cuyes en valles interandinos. XX Reunión ALPA, XXX Reunión APPA-Cusco-Perú. Arch. Latinoam. Prod. Anim. Vol. 15 (Supl. 1) 2007- 229.

2. Chauca Francia de Zaldívar, Lilia. (2007). 
Realidad y perspectiva de la crianza de cuyes en los paises andinos. XX Reunión ALPA, XXX Reunión APPA-Cusco-PerúArch. Latinoam. Prod. Anim. Vol. 15 (Supl. 1) $2007 \cdot 223$.

3. Machahuay Yaranga Jaime. (2012). "Efecto de tres tipos de alimento en la etapa de engorde del cuy (cavia porcellus) en la localidad de Kimbiri - Cuzco - VRAE". Tesis Ingeniero en Ciencias Agrarias Especialidad Zootecnia. Universidad Nacional del Centro del Perú. Satipo, Perú.

4. Ministerio de Agricultura (1997). Estadistica Agraria. INEI.

5. Ordoñez N. Ricardo. (2003). Plan de introducción de la carne de cuy en Lima Metropolitana: Estudio de Mercado y Propuesta Empresarial. Tesis para optar el Gardo de magister en Administracion de Negocios. Pontificia Universidad católica del Peru. Lima.

6. Zumer V.M. (2000). Estudio comparativo de ganancia de peso entre cobayos castrados y no castrados en altura. UNCP Huancayo $78 p$.

7. Owen R.H (2001). El cuy: Recomendaciones para su crianza en el Perú, Lima 18p. 